\title{
Ölabbauende Mikroorganismen in Natur und Technik
}

\author{
Karl Heinz WallhäUsser \\ Mikrobiologisches Untersuchungslabor, Farbwerke Hoechst $A G$, \\ Frankfurt/M.-Höchst
}

\begin{abstract}
Oil decomposing microorganisms in nature and technique. The paper deals with the isolation of microorganisms from oil fields, underground gas reservoirs, sulfur springs, oil tanks, jet fuel tanks, and cutting oils. The role of Desulfovibrio desulfuricans in secondary oil recovery is discussed. Experiments regarding the oil degradation by microorganisms were conducted. Possibilities of "assisting nature" by adding cultures of bacteria to oil polluted waters are discussed.
\end{abstract}

\section{EINLEITUNG}

Der Olabbau durch Mikroorganismen, von welcher Seite man ihn auch betrachtet, spielt heute eine bedeutende Rolle. Während es der Erdölindustrie bei der Ölförderung zunächst darum geht, diese mikrobiellen Einflüsse, die zu einer Porenverengung in der Lagerstätte und zu Korrosionserscheinungen in den Förderanlagen führen können, weitmöglichst auszuschalten, trägt sie sich auf der anderen Seite mit dem Gedanken, mit Hilfe dieser eben noch unliebsamen Mikroorganismen Erdöl zu fraktionieren, um so aus bisher unverwertbaren Rückständen Futtereiweiß zu gewinnen. In den Endprodukten der Erdölraffinerien wiederum sind Mikroorganismen unerwünscht, da sie in den Lagertanks, in den Treibstofftanks der Flugzeuge und in Heizöltanks zu Korrosionserscheinungen (Lochfraß) und Emulsionsbildung führen können. Was aber geschieht nun beispielsweise mit dem Heizöl im Erdboden, wenn ein Ölheizungstank undicht geworden ist oder ein Tankwagen bei einem Unfall ausläuft? Wird das Ol, das sich wie eine Linse im Erdreich ausbreitet, durch die dort vorhandenen Mikroorganismen abgebaut, und welche Zeiträume sind dazu nötig? Dieser Frage wird in der vorliegenden Darstellung nachgegangen. Es muß betont werden, daß wir bei unseren Untersuchungen nur an kleinere Katastrophenfälle gedacht haben, wobei höchstens einige tausend Liter OOl auslaufen. Zur Vernichtung der dabei auftretenden Ollinsen haben wir zwei Möglichkeiten untersucht: (1) die Beimpfung dieser Öllinsen mit ölabbauenden Mikroorganismen unter aeroben und anaeroben Verhältnissen und (2) den Zusatz von Trockenzellen oder Enzympräparaten aus solchen ölabbauenden Mikroorganismen zu der ausgebaggerten Erde, um so (eventuell unter Belüftung) zu einem rascheren Ollabbau zu gelangen. Im folgenden soll über die dazu durchgeführten qualitativen Laboratoriumsversuche berichtet werden. Die quantitative Auswertung der 
noch nicht abgeschlossenen Untersuchungen wird zu einem späteren Zeitpunkt mitgeteilt werden.

\section{MATERIAL UND METHODEN}

Die bei unseren Abbauversuchen eingesetzten Mikroorganismen wurden aus Erdöllagerstätten, Erdgaslagerstätten, Untertagegasspeichern, Schwefelquellen, Raffinerietanks, Düsentreibstofftanks von Flugzeugen, Heizöltanks und aus Bohr- und Schneidölen isoliert. Bei diesen Isolierungen galt unser Hauptaugenmerk jedoch zunächst jenen Organismen, welche durch die von ihnen verursachten Störungen der erdölfördernden Industrie erheblichen Schaden zufügen. Dies führte zwangsläufig zu einer gewissen Einseitigkeit, da die Begleitmikroorganismen vernachlässigt wurden. Erst zu einem späteren Zeitpunkt, bei der Untersuchung von Untertagegasspeichern und Ölspeichern, wurde die an Ort und Stelle vorliegende Mikroflora in ihrer Gesamtheit betrachtet.

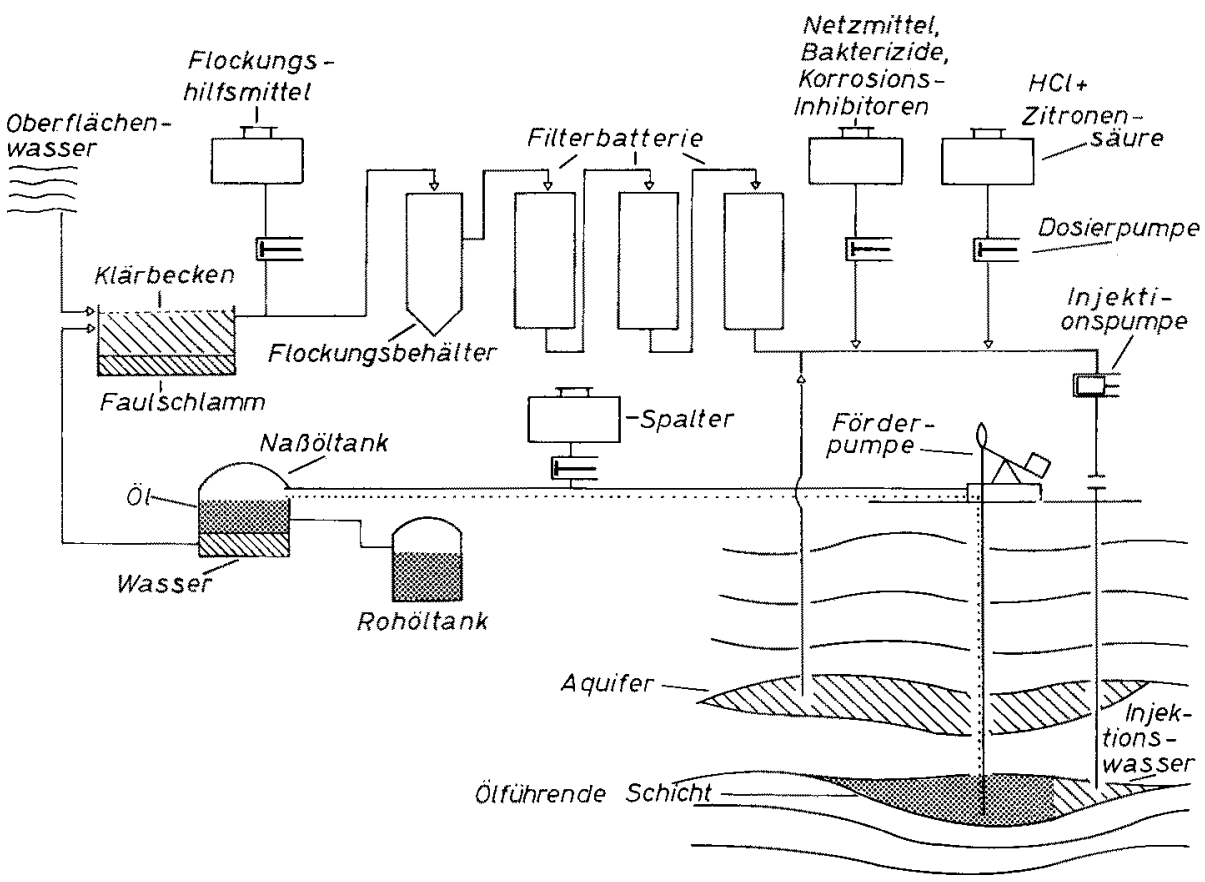

Abb. 1: Schema einer Sekundärförderung zur Gewinnung von Erdöl

BAstin (1926) fand in 28 von 30 untersuchten Wasserproben aus amerikanischen Ólfeldern Desulfovibrio desulfuricans. Sicherlich kommen in diesen Olfeldwässern noch zahlreiche andere Mikroorganismen vor, doch konzentrierte sich das Interesse auf diese anaeroben $\mathrm{H}_{2} \mathrm{~S}$-produzierenden Desulfovibrionen, weil sie für die mikrobielle Korrosion in Erdölförderanlagen, besonders bei der sogenannten Sekundär- 
förderung, verantwortlich sind. Weiterhin können sie in den Olsandlagerstätten zu einer Reduzierung der Permeabilität führen, wobei nach Merк к (1943) die Minderung der Durchlässigkeit bis zu 30\% betragen kann. Das Vorkommen dieser Bakterien in Ollagerstätten berechtigt zur Annahme, daß sie in der Lage sind, Ol abzubauen oder sich von Olbegleitstoffen zu ernähren. Aus deutschen, österreichischen, rumänischen, afrikanischen, arabischen und mittelamerikanischen Ollagerstätten sowie deutschen und österreichischen Untertagegasspeichern und Schwefelquellen wurden in der Folgezeit mehr als 100 Desulfovibrio-Stämme isoliert. Hinsichtlich der Unterdrückungsmöglichkeiten solcher Infektionen wurden einige Stämme näher untersucht (WALlHÄUsSER 1965). Dabei zeigte sich, daß sich diese Organismen hinsichtlich ihrer Hitze- und Kochsalztoleranz ganz erheblich unterscheiden, sich aber verhältnismäßig schnell an

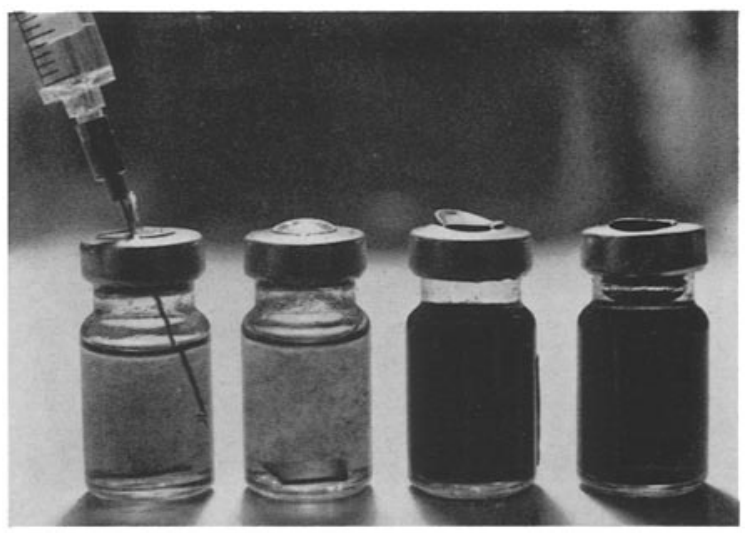

Abb. 2: Testbesteck zum Nadhweis von Desulfovibrio desulfuricans. In die linke Flasche wird die zu untersuchende Wasserprobe mit einer Injektionsspritze eingebracht. Die beiden rechten Flaschen zeigen eine positive Reaktion, das heißt die Bildung von Eisensulfid

die abiotischen Gegebenheiten anpassen können. Dabei ist das Verhalten im Laboratoriumsversuch nicht immer mit den natürlichen Gegebenheiten in Ubereinstimmung zu bringen. So fand WALLHÄUSSER (1965), daß der aus einer Lagerstätte mit einer Temperatur zwischen $120-140^{\circ} \mathrm{C}$ isolierte Stamm Landau im Laborversuch nur $70^{\circ} \mathrm{C}$ toleriert und bei $80^{\circ} \mathrm{C}$ bereits nach 15 Minuten abstirbt.

Nicht immer sind diese Desulfovibrionen autochthon, häufig werden sie mit dem Einpreßwasser bei der Sekundärförderung in die Lagerstärte eingeschleppt (Abb. 1). Eine Bekämpfung einer solchen Lagerstätteninfektion mit Bakteriziden hat jedoch nur dann Erfolg, wenn das Injektionswasser nach Passieren des Klärbeckens und der Filteranlagen behandelt wird.

Das aus Oberflächenwässern (Bächen, Teichen), aus einem über der Ollagerstätte liegenden Aquifer oder aus den Naßöltanks stammende „Injektionswasser“ ist häufig mit Desulfovibrionen infiziert. Rührt man ein Klärbecken auf, in dem die Wässer verschiedener Herkunft meistens vorher vereinigt werden, so beobachtet man die auf die Tätigkeit der Desulfovibrionen zurückzuführende Schwarzfärbung durch Bildung von Eisensulfid. 
Beim Nachweis dieser Mikroorganismen in dem Einpreßwasser stößt man dann auf Schwierigkeiten, wenn es sich um Wasser verschiedener Herkunft mit unterschiedlichem Kochsalzgehalt handelt. Der Kochsalzgehalt wird vor dem Einpressen auf eine für die Lagerstättenverhältnisse optimale Konzentration eingestellt, die sich nach den für das Porenvolumen wichtigen Quellungsverhältnissen der darin vorkommenden Tonbestandteile richtet. Der optimale Kochsalzgehalt schwankt dabei zwischen 4 bis $15 \%$. Wird das Injektionswasser nun mit einem Testbesteck geprüft (Abb. 2), dessen Kochsalzgehalt dem des Injektionswassers von z. B. $6 \% \mathrm{NaCl}$ entspricht, so lassen sich damit die aus dem zugeführten Süßwasser stammenden Desulfovibrionen nicht

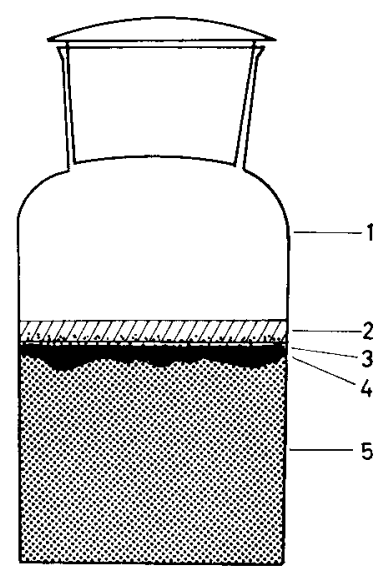

Abb. 3: Laborversuch zur Demonstration des Olabbaues durch Desulfovibrio desulfuricans. In der Wachstumszone tritt infolge der Schwefelwasserstoffbildung und dessen Reaktion mit Eisensulfat eine Schwarzfärbung ein. Ol wird hier als alleinige Kohlenstoffquelle verwertet. $1=$ Glasflasche $(500 \mathrm{ml}), 2=$ Olschicht $(1 \mathrm{~cm}$ hoch $), 3=$ Grenzschicht OI-Sand, $4=$ Schwarzfärbung durch Wachstum von Desulfovibrio desulfuricans, $5=$ Quarzsand (mit Mineralsalzlösung angefeuchtet)

nachweisen. Das Wasser wird dann oft als „einwandfrei“ injiziert, enthält aber Süßwasserstämme, die sich in der Lagerstätte innerhalb kürzester Frist an die neuen Bedingungen, den höheren Kochsalzgehalt, anpassen und später Porenverengung und Lochfraß verursachen. Eine solche Fehlbeurteilung läßt sich nur vermeiden, wenn man mit verschiedenen Testbestecken mit abgestufter Kochsalzkonzentration $(0 ; 1 ; 3 ; 10$ und $15 \%$ ) arbeitet.

Auch bei den von WallhöUsser \& Puchelt (1966) untersuchten Schwefelquellen spielt OOl zum Teil als Nährstoff für die schwefelwasserstoffproduzierenden Desulfovibrionen eine Rolle (Bad Wiessee). Dabei ist jedoch in jedem Falle der Sulfatgehalt des Wassers von ausschlaggebender Bedeutung und tritt als begrenzender Faktor auf. Unter Bakterizidbehandlung niedergebrachte Bohrungen zur Erschließung von Untertagegasspeichern haben in den beiden letzten Jahren gezeigt (WALLHÄUSSER, in Vorbereitung), daß nicht immer "sterile Speicher“ erschlossen werden können, sondern daß häufig, insbesondere wenn in dem Lagerstättenwasser Spuren von Erdöl oder Erdgas und Sulfat vorhanden sind, bereits Desulfovibrionen und 2 bis 3 weitere 
Bakterien in einer Gesamtkeimzahl bis zu 1 Million/ml anzutreffen sind. Es muß angenommen werden, daß es sich hier um Mikroorganismen handelt, die durch Verwerfungszonen in die etwa 400 bis $600 \mathrm{~m}$ unter der Erdoberfläche im Buntsandstein und Keuper liegenden, für die Untertagegasspeicherung vorgesehenen Schichten eingewandert sind.

Neben Desulfovibrio wurden in den verschiedensten Lagerstätten und Oiltanks sowie in Bohr- und Schneidölen Arten der Gattungen Pseudomonas, Micrococcus (vermutlich Micrococcus paraffinae), Sarcina und Hormodendron im Düsentreibstoff nachgewiesen.

In den vorliegenden Untersuchungen haben wir uns zunächst auf den Olabbau durch Desulfovibrio desulfuricans-Stämme und Hormodendron spec. beschränkt. Für die Durchführung der Versuche wurden praxisnahe Bedingungen gewählt (Abb. 3).

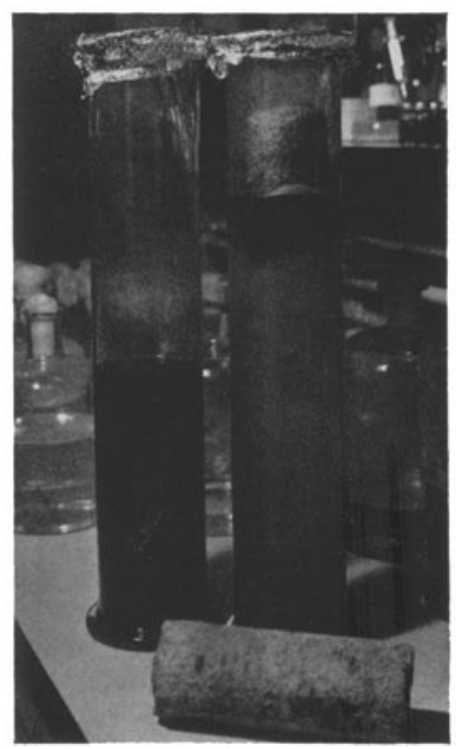

Abb. 4: Das in einem Bohrkern enthaltene Ol dient hier als Kohlenstoffquelle für Desulfovibrio desulfuricans. Die Vergleichskultur links verwendet Calciumlactat als C-Quelle

Glasflaschen $(500 \mathrm{ml})$ wurden mit Quarzsand gefüllt und nachher mit einer Mineralsalzlösung $\left(0,2 \mathrm{~g} \mathrm{MgSO}_{4} \cdot 7 \mathrm{H}_{2} \mathrm{O} ; 0,1 \mathrm{~g} \mathrm{Fe}\left(\mathrm{NH}_{4}\right)_{2}\left(\mathrm{SO}_{4}\right)_{2} \cdot 6 \mathrm{H}_{2} \mathrm{O} ; 0,1 \mathrm{~g} \mathrm{Fe} \mathrm{SO} \mathrm{S}_{4}\right.$. $7 \mathrm{H}_{2} \mathrm{O} ; 1000 \mathrm{ml}$ Aqua dest; $\left.\mathrm{pH} 7,2\right)$ bis etwa $1 \mathrm{~cm}$ unter der Oberfläche aufgefüllt (Abb. 3). Bei den Desulfovibrio-Versuchen wurden dem Sand 20 Eisenplättchen (je $1 \mathrm{~cm}^{2}$ ) zugesetzt. Anschließend wurde mit einer mehrere Tage ( 3 bis 5 Tage) alten Kultur von Desulfovibrio desulfuricans-Stämmen beimptt und der Ansatz dann mit $25 \mathrm{ml}$ Ol überschichtet. Auf diese Weise wurde der Abbau von Rohöl und Heizöl durch 20 verschiedene Desulfovibrio-Stämme untersucht.

Um die Bedeutung großer Berührungsflächen zwischen OI und Wasser zu demonstrieren, wurden in einer weiteren Versuchsserie ölhaltige Bohrkerne in einen Meß- 
zylinder in die obengenannte, lediglich mit einem Zusatz von 0,2\% Agar versehene Mineralsalzlösung eingehängt (Abb. 4). Die Beimpfung erfolgte auch hier mit Desulfovibrio desulfuricans. Als Vergleich diente ein Ansatzgefäß, in dem Calciumlactat als C-Quelle diente.

Der aus Düsentreibstoff isolierte Pilz Hormodendron spec. entwickelt sich, wie Abbildung 5 zeigt, ebenfalls an der Berührungszone Treibstoff - Wasser, wobei später das Mycel in die Wasserphase absinkt. In so angelegten Standkulturen, in denen die Wasserphase (pH 5,5) auf 1 Liter $2 \mathrm{~g} \mathrm{NaNO}_{3}, 1 \mathrm{~g} \mathrm{KH}_{2} \mathrm{PO}_{4}, 0,5 \mathrm{~g} \mathrm{MgSO}_{4} \cdot 7 \mathrm{H}_{2} \mathrm{O}$, $0,01 \mathrm{~g} \mathrm{FeSO}_{4}$ and $0,5 \mathrm{~g} \mathrm{KCL}$ enthielt, entwickelte sich der Pilz sehr langsam. Als Kohlenstoffquelle diente hierbei Düsentreibstoff. Sichtbares Wachstum trat in diesen Ansätzen erst nach 3 bis 4 Wochen auf.

Zur Ergänzung dieser Versuche wurden von diesem aerob wachsenden Pilz Schüttelkulturen in $300 \mathrm{ml}$ Erlenmeyerkolben mit $80 \mathrm{ml}$ der oben genannten Mineralsalzlösung und unter Zusatz von 1 bis $5 \%$ Düsentreibstoff, Heizöl bzw. Rohöl angelegt. Der Olabbau wurde hierbei nicht mit chemischen Methoden bestimmt, sondern das Mycel wurde nach 10 Tagen abgeschleudert, mit Methylenchlorid gewaschen, 24 Std. bei $100^{\circ} \mathrm{C}$ getrocknet, und anschließend das Trockengewicht bestimmt. Gewaschenes Mycel aus diesen Ansätzen wurde anschließend zu „ölverschmutztem Sand“, entsprechend dem Versuchsansatz in Abbildung 3, zugesetzt und auch hier der Olabbau über einen längeren Zeitraum verfolgt, wobei in einigen Versuchen der Sand (Erdboden) mit Hilfe von Sonden belüftet wurde.

\section{ERGEBNISSE UND DISKUSSION}

In unseren Versuchsreihen dienten Rohöl, Heizöl bzw. Düsentreibstoff oder die darin enthaltenen Produkte als einzige Kohlenstoffquelle. Bei Ausnutzung dieser C-Quellen mußte in der auf Abbildung 3 gezeigten Versuchsanordnung, bei der Entwicklung von Desulfovibrio desulfuricans, durch dessen Schwefelwasserstoffbildung eine Schwarzfärbung (Eisensulfidbildung) auftreten. In der Regel beobachtete man diese Reaktion nach 2 bis 3 Wochen im Bereich der Berührungszone zwischen OI und Mineralsalzlösung. Hätten bei diesen Versuchen in den Olen enthaltene wasserlösliche Produkte eine Rolle gespielt, so wären diese sicherlich auch in den tieferen Bereich der Sandkultur diffundiert und es hätte dann auch dort zu einer Schwarzfärbung kommen müssen. Dies wurde jedoch in keinem Falle beobachtet. Der Olabbau erfolgte in diesen Ansätzen nur sehr langsam, und selbst nach einem Jahr war keine nennenswerte $\mathrm{Ab}$ nahme der überstehenden Ölschicht festzustellen.

Wesentlich rascher stellte sich die Entwicklung von Desulfovibrio desulfuricans bei der auf Abbildung 4 gezeigten Versuchsanordnung ein. Wir führen diese Tatsache auf die größere Berührungsfläche $z w i s c h e n$ OI und Wasser, also die feinere Verteilung des Ols zurück. Dieser Versuch demonstriert zugleich die Verhältnisse bei der Sekundärförderung von Erdöl aus einer Olsandlagerstätte. Durch die großen Berührungsflächen zwischen OI und Wasser im Porenraum der Lagerstätte kommt es dort zu einer raschen Vermehrung von Desulfovibrio desulfuricans. Ganz anders liegen die Verhältnisse bei einer "Ólblase“ oder einer „Ollinse“, wie sie beispielsweise beim Auslaufen eines Ol- 
tanks im Erdboden entstehen können. Hier ist die Angriffsfläche für die ölabbauenden Mikroorganismen sehr klein, und der Abbau vollzieht sich über lange Zeiträume. Wäre es anders, so hätten sich unsere Olvorräte in den kompakten Lagerstätten wohl kaum über Jahrmillionen halten können. Dieser Versuch zeigt zugleich, daß bei der Lagerung von Kohlenwasserstoffen in Untertagespeichern (zur Vorratshaltung) dem Kavernenspeicher (kompakte Lagerung) vor dem Aquifer-Speicher (Lagerung im porösen, teilweise wassergefüllten Raum) der Vorrang einzuräumen ist.

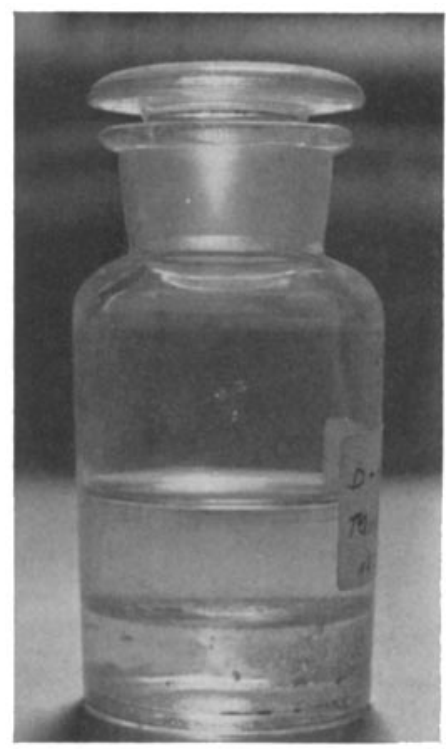

Abb. 5: An der Grenzschicht zwischen Düsentreibstoff und Wasser entwickelt sich der Pilz Hormodendron. Das Pilzmycel sammelt sich stets nur in der Wasserphase, nie in dem Treibstoff an

Auch die Ölabbau-Versuche im Quarzsand-Gefäß mit zugesetztem, gewaschenem Mycel von Hormodendron spec. verliefen unbefriedigend. Zugesetzte Emulgatoren beschleunigten zwar zunächst den Abbau, wurden aber dann, wie wir es vorher schon bei Bohr- und Schneidölen beobachtet hatten, sehr rasch von den Mikroorganismen abgebaut (Abb. 5). Über den Olabbau durch Hormodendron spec. und Streptomyceten, jeweils 10 Stämmen der grauversporenden griseus-Gruppe und der grünversporenden prasinus-Gruppe, in Schüttelkulturen wird zu einem späteren Zeitpunkt an anderer Stelle berichtet werden.

\section{ZUSAMMENFASSUNG}

1. Praxisnahe Versuche mit ölabbauenden Mikroorganismen, insbesondere Stämmen von Desulfovibrio desulfuricans und Hormodendron spec., die aus verschiedenen Ollagerstätten und Öltanks isoliert wurden, haben gezeigt, daß der Abbau einer geschlossenen Ơlschicht nur sehr langsam erfolgt. 
2. Nach den gewonnenen Ergebnissen dürfte es unwahrscheinlich sein, eine beispielsweise durch Auslaufen eines Oltanks im Boden entstandene „Ollinse" durch Zusatz von geeigneten, ölabbauenden Mikroorganismen innerhalb weniger Tage zu beseitigen, um so die Gefahr der Olverschmutzung des Trinkwassers zu bannen.

\section{ZITIERTE LITERATUR}

Bastin, E. S., 1926. The problem of the natural reduction of sulphates. Bull. Am. Ass, Petrol. Geol. 10, 1270.

Merkt, E. E., 1943. Thesis, Austin, Univ. of Texas,

Wat.thäUSSER, K. H., 1965. Mikrobiologische Untersuchungen über Flutwässer bei der Erdölgewinnung. Erdöl Koble 18, 357-360.

- 1968. Untersudungen über die Tätigkeit von Mikroorganismen in Untertagegasspeichern. Erdäl Kohle. (In Vorber.)

- \& Puchelт, H., 1966. Sulfatreduzierende Bakterien in Schwefel- und Grubenwässern Deutschlands und Osterreichs. Contr. Mineral. Petrol 13, 12-30. 